\title{
Vitiligo patients show significant up-regulation of aryl hydrocarbon receptor transcription factor*
}

Farinaze Behfarjam ${ }^{1}$
Zohreh Jadali $^{2}$

\section{DOI: http://dx.doi.org/10.1590/abd1806-4841.20187183}

\begin{abstract}
IL-22 has been implicated in the pathogenesis of vitiligo. However, the role of aryl hydrocarbon receptor transcription factor that acts as a master regulator of IL-22-producing Th22 cells is not fully understood. The goal of this study was to investigate the expression pattern of aryl hydrocarbon receptor in peripheral blood mononuclear cells of patients with vitiligo and in normal controls. Transcript levels were determined by a reverse transcription quantitative real-time polymerase chain reaction. Aryl hydrocarbon receptor mRNA expression was drastically increased in patients with vitiligo compared to healthy controls $(\mathrm{P}=$ 0.000). Th22 cells may contribute to abnormal immune responses underlying vitiligo.
\end{abstract}

Keywords: Autoimmunity; T-Lymphocytes; Vitiligo

Many findings point toward a central role for the immune system in vitiligo pathogenesis. Unfortunately, the exact mechanism(s) that allows the immune system to recognize and kill skin melanocytes remains unknown. Nonetheless, recent investigations indicate a marked perturbation in CD4+ T helper (Th) cells homeostasis in vitiligo patients.

Th22 cell is a newly described subset of CD4+ T cells. As implied by the name, they are the most important source of IL-22, and aryl hydrocarbon receptor (AhR) is a critical transcription factor (TF) for their development. ${ }^{2}$ Such dependencies on the recruitment of specific TF can provide a valuable approach for molecular distinction of Th22 subpopulation from other important sources of IL-22 such as Th17 cells. Therefore, the purpose of this study was to examine the mRNA expression levels of Th22-specifying transcription factor AhR in vitiligo patients.

After blood draw and peripheral blood mononuclear cell (PBMC) separation by Ficoll-hypaque, total cellular RNA was extracted and cDNA was synthesized. cDNA amplification was conducted by qRT-PCR using SYBR Premix EX Taq II (Takara, Japan).

The study group consisted of 22 patients with inactive vitiligo (11 men and 11 women) and 22 healthy controls ( 8 men and 14 women). The clinical forms were generalized vitiligo $(n=17)$, vitiligo universalis $(n=4)$, and segmental vitiligo $(n=1)$. All patients were new cases who were not being treated for their diseases or were medication-free for at least three weeks prior to the study. The mean ages of the patients and controls were $33.91 \pm 9.86$ and $39.91 \pm 14.52$ years, respectively.

As shown in Figure 1, greater AhR expression (7.03 \pm 1.72$)$ was found in the PBMCs of patients as compared to healthy controls (9.24 \pm 1.99$)(P=0.000)$. The difference in AhR expression between patients and controls was not affected by age, gender, or disease duration.

Some evidence indicates that $\mathrm{T}$ cells are indispensable for the immune-mediated destruction of melanocytes. However, the exact contribution of the different $\mathrm{CD} 4+\mathrm{T}$ cell subpopulations in disease pathogenesis is incompletely understood.

In recent years, considerable attention has been paid to Th22 cells that have distinct gene expression profiles in immune response. They express skin homing chemokine receptors, allowing them to migrate into skin. ${ }^{3}$ Furthermore, the skin has been identified as one of the main targets of IL-22, a major product of Th22 cells. This is due to the expression of IL-22 receptor on the surface of critical cellular components of the skin barrier. ${ }^{4}$ Therefore, it is thought that Th22 cell is a major player in skin homeostasis. However, under specified conditions, their aberrant function can result in the development of undesirable processes that are implicated in the patho-

\footnotetext{
Received on 03.04.2017.

Approved by the Advisory Board and accepted for publication on 09.07.2017.

* Work performed at the Tehran University of Medical Sciences.

Financial support: None.

Conflict of interest: None.

Clinical Genetics Department, National Institute of Genetic Engineering and Biotechnology Tehran, Iran.

Department of Immunology, School of Public Health, Tehran University of Medical Sciences, Tehran, Iran.
}

MAILING ADDRESS:

Zohreh Jadali

E-mail: zjadali@yahoo.co.uk;

zjadali@razi.tums.ac.ir 


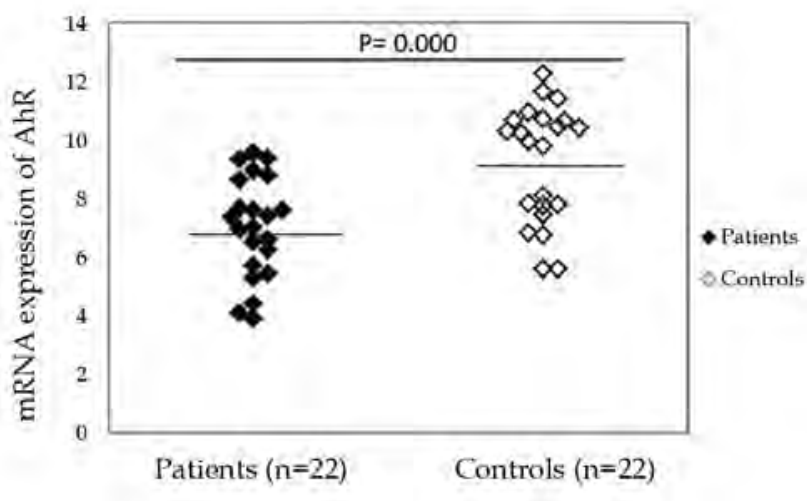

FiguRE 1: Detection of AhR mRNA in peripheral blood mononuclear cells from patients with vitiligo and healthy persons by reverse transcription-quantitative polymerase chain reaction. AhR mRNA levels were significantly higher in patients than normal persons. Please note that a higher $\Delta \mathrm{Ct}$ value corresponds to a comparably lower expression level

genesis of different autoimmune diseases including skin-related autoimmune diseases.

The effector functions of Th22 cells are mainly mediated by their secreted cytokines, especially IL-22. However, IL-22 production is not confined to the Th22 subset, with a variety of known IL$22-$ producing cell types. Thus, the quantification of Th22 specific TF provides a better understanding of the cellular sources of IL-22. This procedure could help explain the underlying pathogenic mechanisms of the disease.

The current study indicates a statistically significant difference in AhR expression between patients and control groups, suggesting that this TF may have a potential role in disease pathogenesis.

These results are not consistent with the findings of Wang et al., who showed decreased expression of AhR in the PBMCs of vitiligo patients. ${ }^{5}$ In contrast, Elela et al. showed significantly increased

\section{REFERENCES}

1. Boniface $K$, Taïeb A, Seneschal J. New insights into immune mechanisms of vitiligo. G Ital Dermatol Venereol. 2016;151:44-54.

2. Esser C, Bargen I, Weighardt H, Haarmann-Stemmann T, Krutmann J. Functions of the aryl hydrocarbon receptor in the skin. Semin Immunopathol. 2013:35:677-91.

3. Fujita H. Role of IL-22 in the pathogenesis of skin diseases. Nihon Rinsho Meneki Gakkai Kaishi. 2012;35:168-75.

4. Witte E, Witte K, Warszawska K, Sabat R, Wolk K. Interleukin-22: a cytokine produced by T, NK and NKT cell subsets, with importance in the innate immune defense and tissue protection. Cytokine Growth Factor Rev. 2010;21:365-79.

5. Wang X, Li K, Liu L, Shi Q, Song P, Jian Z, et al. AHR promoter variant modulates its transcription and downstream effectors by allele-specific AHR-SP1 interaction functioning as a genetic marker for vitiligo. Sci Rep. 2015;5:13542.

6. Elela MA, Hegazy RA, Fawzy MM, Rashed LA, Rasheed H. Interleukin 17, interleukin 22 and FoxP3 expression in tissue and serum of non-segmental vitiligo: a casecontrolled study on eighty-four patients. Eur J Dermatol. 2013;23:350-5.
IL-22 levels in sera and skin biopsies of patients compared with controls. ${ }^{6}$ In our opinion, different factors are the sources of these controversies. The first and most important one is the extensive role of AhR in immune regulation. These regulatory effects are mediated through its influence on T-cell differentiation and cytokine milieu. Interestingly, all skin cells express AhR. ${ }^{2}$ It is also important to note that AhR is essential for proliferation or differentiation of melanocytes and regulation of melanogenesis. ${ }^{7}$

Secondly, AhR is a ligand-activated TF and provides a link between environmental triggers and immune responses. The effects of these ligand-receptor interactions on the immune system are determined by different factors, which include the type and activation status of cell types, the agonistic or antagonistic properties of ligands, and the cytokine millieu. ${ }^{8}$ Therefore, it is difficult to determine the exact effect that AhR activation exerts on immune system in normal and disease conditions.

The results of the present study demonstrated an increase in mRNA expression of AhR. Thus, transcriptional activation of AhR and $A h R$ target genes may be implicated in vitiligo pathogenesis. The expression pattern of $\mathrm{AhR}$ is particularly interesting in vitiligo patients since the promoter region of several melanogenic factors contains an AhR responsive element that could be under the control of AhR. ${ }^{9}$ Moreover, down-regulation of AhR and AhR-regulated genes was detected in the epidermis layer of patients with vitiligo. ${ }^{10}$

Although our results highlight the possible importance of AhR in vitiligo, they also address one of the main limitations of this study: AhR expression was not evaluated in normal skin melanocytes from vitiligo patients. A small sample size is another limitation of this study for restricting the interpretation of the results. Consequently, more studies with larger samples are needed to explore the nature of gene-gene or gene-environment interaction in vitiligo patients.

This study indicates that increased AhR expression occurs in the PBMCs of vitiligo patients, which suggests a potential association between Th22 cells and the disease. Nonetheless, the exact role of these cells in disease pathogenesis is questionable and further studies are required to fully elucidate the role of these cells in vitiligo. $\square$

7. Luecke S, Backlund M, Jux B, Esser C, Krutmann J, Rannug A. The aryl hydrocarbon receptor (AHR), a novel regulator of human melanogenesis. Pigment Cell Melanoma Res. 2010;23:828-33

8. Cella M, Colonna M. Aryl hydrocarbon receptor: Linking environment to immunity Semin Immunol. 2015;27:310-4.

9. Frericks M, Burgoon LD, Zacharewski TR, Esser C. Promoter analysis of TCDDinducible genes in a thymic epithelial cell line indicates the potential for cell-specific transcription factor crosstalk in the AhR response. Toxicol Appl Pharmacol. 2008;232:268-79

10. Schallreuter KU, Salem MA, Gibbons NC, Maitland DJ, Marsch E, Elwary SM, et al. Blunted epidermal L-tryptophan metabolism in vitiligo affects immune response and ROS scavenging by Fenton chemistry, part 2: Epidermal H2O2/ONOO(-)-mediated stress in vitiligo hampers indoleamine 2,3-dioxygenase and aryl hydrocarbon receptor-mediated immune response signaling. FASEB J. 2012;26:2471-85.

How to cite this article: Behfarjam F, Jadali Z CN. Vitiligo patients show significant up-regulation of aryl hydrocarbon receptor transcription factor. An Bras Dermatol. 2018;93(2):302-3. 